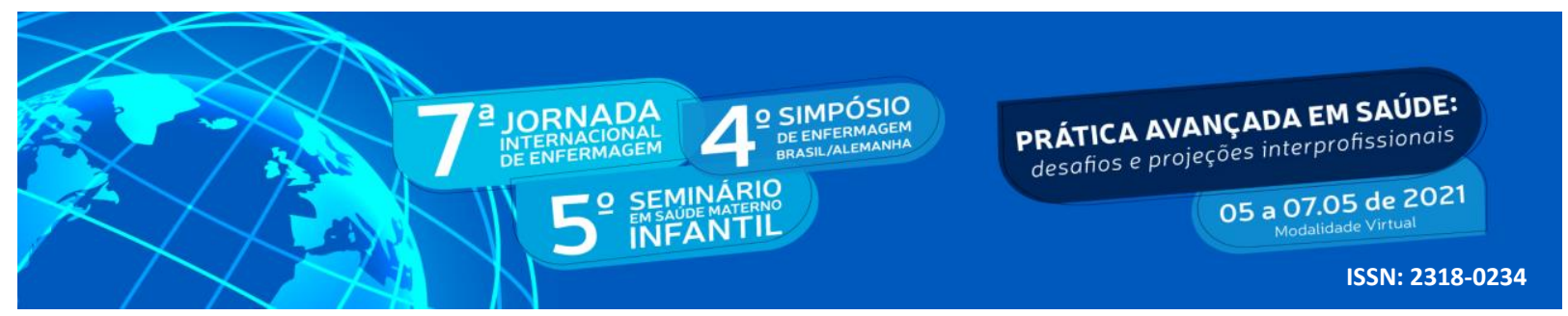

DOI: http://doi.org/10.48195/jie2021-071

\title{
RISCO DE CONTAMINAÇÃO MICROBIOLÓGICA E GESTÃO DA FARDA CLÍNICA NOS CUIDADOS DE ENFERMAGEM À CRIANÇA
}

\author{
Ana Laura Pereira'; ${ }^{1}$ Inês Abreu ${ }^{2}$; Rafaela Oliveira ${ }^{2}$; João Graveto ${ }^{3}$;Lurdes Lomba ${ }^{4}$ \\ RESUMO
}

Estudo quantitativo, descritivo-correlacional transversal, com uma amostra de 24 enfermeiros de pediatria, aos quais foram aplicados questionários e realizadas colheitas microbiológicas das fardas clínicas, objetivando verificar a relação entre os cuidados de enfermagem de contacto da farda com a criança, a gestão da farda pelos enfermeiros e os microrganismos nela presentes. Foram encontradas nas fardas clínicas: Staphylococcus Aureus; outras bactérias do grupo Staphylococcus; do grupo dos bacilos; do grupo Streptococcus e Acinetobacter baumannii, com maior percentagem nas regiões do abdómen e tórax à esquerda. Verificou-se existir relação entre os cuidados de enfermagem e o risco de contaminação microbiológica no método de canguru e colo para conforto, transporte ou contenção. A gestão da farda clínica exige cuidados especiais no seu tratamento e a necessidade de uniformizar práticas e adequar comportamentos que minimizem o risco de contaminação microbiológica. Recomenda-se o uso de Equipamentos de Proteção Individual e mudança diária da farda.

Palavras-chave: Contaminação; Criança; Enfermeiros; Infeção; Vestuário.

\begin{abstract}
Quantitative, descriptive-correlational cross-sectional study, with a sample of 24 pediatric nurses to whom questionnaires were applied and microbiological samples of the clinical uniforms were collected, with the purpose of verifying the relationship between the nursing care provided by the contact between the uniform and the child, the management of the uniform by nurses, and the microorganisms present in it. The following organisms were found in the clinical uniforms: Staphylococcus Aureus; other bacteria of the Staphylococcus group; the bacilli group; the Streptococcus group, and Acinetobacter baumannii, with a higher percentage in the abdomen and left thorax regions. A relationship was found between nursing care and the risk of microbiological contamination in the kangaroo method and holding for comfort, restraint and transportation. The management of the clinical uniform requires special care in its handling and the need to standardize practices and adapt behaviors that minimize the risk of microbiological contamination. The use of Personal Protection Equipment and daily change of uniform is recommended.
\end{abstract}

Key Words: Contamination; Child; Nurses; Infection; Uniform.

\footnotetext{
${ }^{1}$ CHUC. E-mail: analaurapereira@ outlook.pt

2 Escola Superior de Enfermagem de Coimbra. E-mail: inesdaluzabreu@gmail.com; rafaelaoliv1514@gmail.com

${ }^{3}$ Co-Orientador. UICISA:E. Escola Superior de Enfermagem de Coimbra. E-mail: jgraveto@esenfc.pt

4 Orientadora. UICISA:E. Escola Superior de Enfermagem de Coimbra. E-mail: mlomba@esenfc.pt
} 


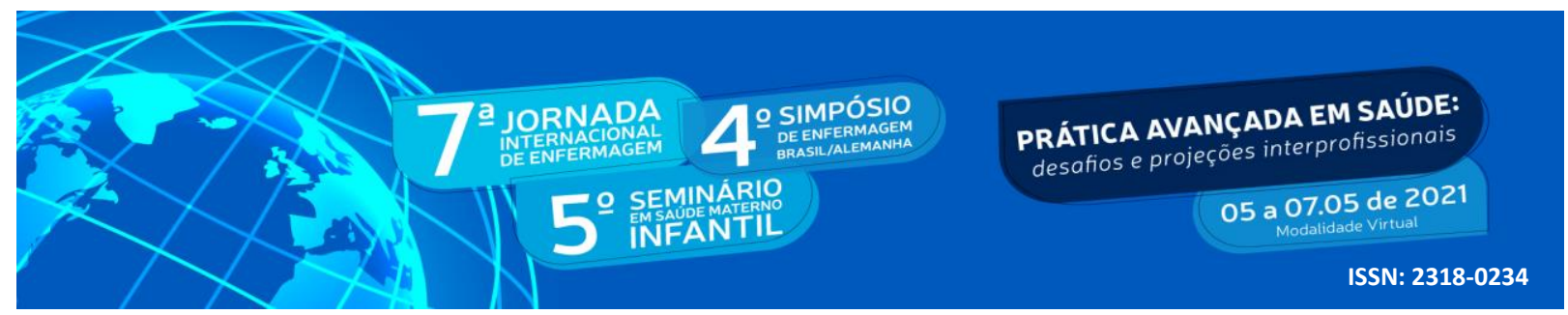

\section{INTRODUÇÃO}

As infeções nosocomiais associadas aos cuidados de saúde (IACS) são um problema de saúde pública, tanto em Portugal, como no resto do mundo, afetando tanto países desenvolvidos como em desenvolvimento (Carvalho \& Marques, 1999; Jacinto, 2019). A segurança do paciente e a prestação de cuidados de qualidade devem ser prioridades dos profissionais de saúde, sendo a prevenção e o controlo de infeções partes fundamentais dos cuidados, o que leva à necessidade de medidas preventivas de disseminação de infeções (Gonçalves, 2014). Segundo Anderson et al. (2017), os microrganismos patogénicos são espalhados nos hospitais pelos profissionais de saúde através de cuidados que envolvem o contato com os pacientes, familiares, ambiente do paciente e outros profissionais. A utilização de fardas clínicas apresenta vantagens, como a promoção da segurança e proteção do profissional e do doente, conforto e estética do profissional, para além de ser um elemento de identidade corporativa. No entanto, as fardas são rotineiramente contaminadas durante os cuidados de enfermagem e funcionam como meio de transmissão de microrganismos para outros utentes, familiares e outros profissionais, designando-se este processo como o triângulo de transmissão.

Em enfermagem de saúde infantil e pediatria o contacto da criança com a farda clínica é inevitável e o risco de contaminação das fardas clínicas é elevado, uma vez que os cuidados prestados à criança incluem o uso de técnicas que implicam um contacto físico direto da criança com a farda clínica, como o uso do método canguru ou o colo para aconchego. Dado que a farda acompanha diariamente o enfermeiro na prestação de cuidados, a sua incorreta gestão pode ter consequências negativas na incidência de IACS e na segurança dos recém-nascidos (RN) e das crianças (Oliveira, Silva, \& Garbaccio, 2012). No contexto pediátrico, o controlo da infeção assume um papel fulcral e complexo, tendo em conta as características específicas desta população e os fatores que contribuem para a transmissão da infeção (Siegel, Rhinehart, Jackson \& Chiarello, 2007). A vulnerabilidade à doença do RN e da criança pequena deve-se a características específicas desta população, como o sistema imunitário imaturo, limitada capacidade de defesa contra agressões externas e maior proximidade com os profissionais de saúde, sendo estes mais suscetíveis às infeções e aos microrganismos patogénicos do que as crianças maiores e adultos (Siegel, Rhinehart, Jackson, \& Chiarello, 2007). 


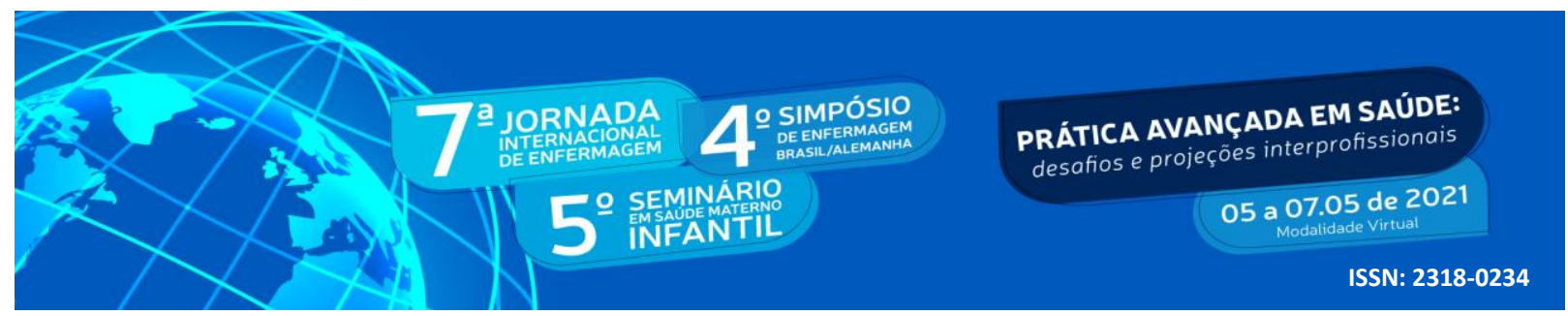

\section{OBJETIVO}

Objetiva-se verificar a relação existente entre os cuidados de enfermagem, em que ocorre contacto da farda clínica com o RN e a criança pequena (até 3 anos), com o modo como é efetuada a gestão da farda pelos enfermeiros e com os microrganismos presentes na mesma.

\section{METODOLOGIA}

Estudo quantitativo, descritivo-correlacional transversal. Coleta de dados realizada de setembro a outubro de 2018, numa Unidade de Cuidados Neonatais (UCN) e serviço de pediatria de um Hospital de Portugal. A amostra é composta por 24 enfermeiros que prestam cuidados a RN e crianças até aos 3 anos, que se apresentaram disponíveis a participar no estudo de forma voluntária e que cumpriam os critérios de inclusão do estudo.

A coleta de dados incluiu um inquérito por questionário e colheitas de amostras microbiológicas, recorrendo à técnica da zaragatoa humedecida nas fardas clínicas dos enfermeiros. Questionário e colheita de amostras microbiológicas foram realizadas no mesmo dia e no mesmo turno aos enfermeiros e encaminhadas para análise laboratorial.

O questionário, anónimo e individual, permitiu a caracterização demográfica da amostra e identificar os cuidados de enfermagem pediátricos, na sua diversidade e frequência, que implicam o contacto direto da criança com a farda clínica; verificar como os enfermeiros gerem a farda clínica; equipamentos de proteção individual utilizados, frequência de substituição da farda e local da sua higienização.

As colheitas de amostras microbiológicas pretendiam identificar os microrganismos existentes na farda clínica e que constituem risco microbiológico para a criança. Foram colhidas amostras nas regiões abdominal (RA), tórax à esquerda (TE) e tórax à direita (TD). Evidências científicas referem ser estas as zonas de maior contato com a criança pequena.

\section{RESULTADOS E DISCUSSÃO}

Do total da amostra, 13 enfermeiros são do serviço de Pediatria e 11 são da UCN. Metade dos enfermeiros têm idade igual ou superior a 50 anos e 54,2\% têm experiência profissional superior a 26 anos. Todos os enfermeiros participantes no estudo são destros. 


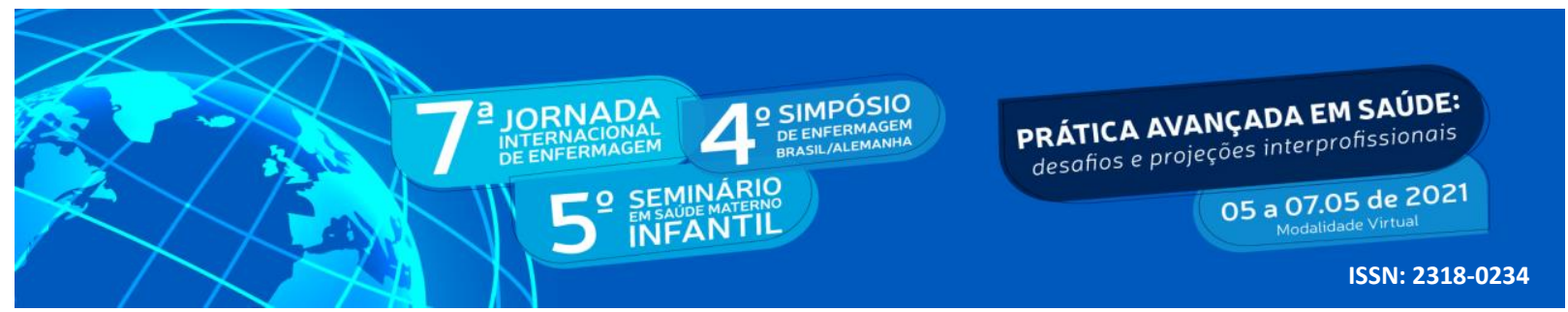

Na tabela 1 observam-se os cuidados de enfermagem mais prestados pelos enfermeiros e a quantidade de vezes em que houve contacto da criança com a farda clínica.

\begin{tabular}{|c|c|c|c|c|c|}
\hline \multirow{3}{*}{$\begin{array}{c}\text { Cuidados em que há } \\
\text { contacto direto da } \\
\text { criança com a farda } \\
\text { clínica }\end{array}$} & \multicolumn{3}{|c|}{ Frequência com que os cuidados foram prestados } & \multirow{2}{*}{\multicolumn{2}{|c|}{$\begin{array}{c}\mathbf{N}^{\circ} \text { e } \% \text { de } \\
\text { enfermeiros que } \\
\text { prestaram cuidados }\end{array}$}} \\
\hline & \multirow{2}{*}{$\begin{array}{c}\mathbf{1 x} \text { por turno } \\
1\end{array}$} & \multirow{2}{*}{$\begin{array}{c}\mathbf{2 - 5 x} \text { por turno } \\
11\end{array}$} & \multirow{2}{*}{$\frac{\mathbf{+ 5 x} \text { por turno }}{12}$} & & \\
\hline & & & & 24 & $100 \%$ \\
\hline $\begin{array}{c}\text { Administração } \\
\text { terapêutica }\end{array}$ & 8 & 8 & 4 & 20 & $83,3 \%$ \\
\hline Outros cuidados & 7 & 5 & 4 & 16 & $66,7 \%$ \\
\hline Cuidados de higiene & 5 & 6 & 4 & 15 & $62,5 \%$ \\
\hline Alimentação & 2 & 2 & 6 & 10 & $41,6 \%$ \\
\hline Colo & 5 & 2 & 1 & 8 & $33,3 \%$ \\
\hline Transferências & 5 & 3 & 0 & 8 & $33,3 \%$ \\
\hline Método canguru & 1 & 4 & 3 & 8 & $33,3 \%$ \\
\hline
\end{tabular}

Tabela 1 - Distribuição dos cuidados de enfermagem em que há contacto da criança com a farda clínica dos enfermeiros

Quanto à gestão da farda clínica, apenas 14 dos 24 enfermeiros referem que a farda que tinham vestida no dia da colheita de dados era de uso diário. Os restantes 10 enfermeiros já tinha usado a farda em três turnos. Os motivos de substituição da farda são a sujidade visível para 24,2\% dos enfermeiros, padrão pessoal de mudança de farda para $25 \%$ e contaminação com fluidos orgânicos para $20,8 \%$ dos enfermeiros. Já $41,7 \%$ dos enfermeiros substituíram a farda clínica por mais do que um dos motivos descritos. Neste contexto, Tipple, Pereira, Hayashida, Moriya e Souza (2003) e Bearman et al. (2014), reforçam que os profissionais de saúde devem trocar a farda clínica diariamente e/ou sempre que ocorra algum contato com secreção ou sangue. Estas recomendações foram igualmente estudadas por Valadares (2017), que verificaram uma relação causal entre a contaminação das fardas, o seu tempo de uso e atividades desenvolvidas, tendo sido verificado um aumento de $154 \%$ no número de colónias entre o início e o final do turno.

No que concerne ao local de higienização da farda clínica, 58,3\% da amostra refere lavar a farda no Hospital e 37,5\% lavam a farda no domicílio. O motivo apontado para não lavar a farda no Hospital foi "o receio" de não ter a farda para o turno seguinte. Segundo as diretrizes estabelecidas no Programa Nacional de Controlo da Infeção de 2006, a farda clínica dos profissionais deve ser tratada preferencialmente na lavandaria da instituição de saúde e de acordo com as Precauções Básica de Controlo da Infeção relativas ao manuseamento de roupa segura (Direção-Geral da Saúde, 2013). Neste sentido o International Scientific Forum on 


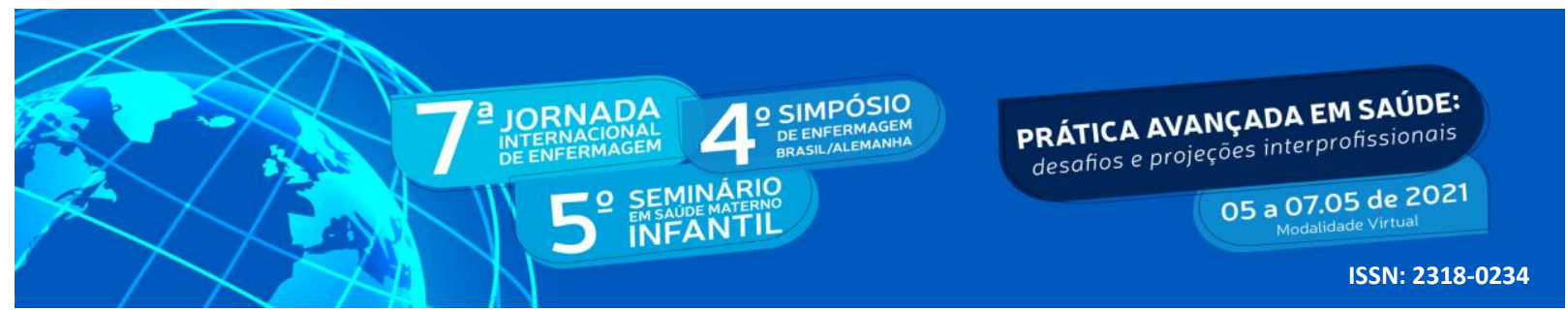

Home Hygiene Information (2013) categoriza a higienização da farda no domicílio como um procedimento de elevado risco.

Quanto aos Equipamentos de Proteção Individual (EPI), a tabela 2 mostra que a sua utilização na prestação de cuidados à criança não é efetuada pela maioria dos Enfermeiros.

\begin{tabular}{|l|c|c|c|c|}
\hline $\begin{array}{c}\text { Cuidados prestados } \\
\text { que requerem: }\end{array}$ & \multicolumn{2}{|c|}{$\begin{array}{c}\mathbf{N}^{\mathbf{0}} \text { e \% de enfermeiros que } \\
\text { prestaram os cuidados }\end{array}$} & \multicolumn{2}{c|}{$\begin{array}{c}\mathbf{N}^{\mathbf{0}} \text { e \% de enfermeiros que utilizaram } \\
\text { EPI na prestação dos cuidados }\end{array}$} \\
\hline Técnica asséptica & 20 & $83,3 \%$ & 6 & $30 \%$ \\
\hline $\begin{array}{l}\text { Contacto direto com a } \\
\text { criança pequena }\end{array}$ & 17 & $70,8 \%$ & 2 & $8,3 \%$ \\
\hline $\begin{array}{l}\text { Risco de exposição a } \\
\text { matéria orgânica }\end{array}$ & 23 & $95,8 \%$ & 6 & $39,1 \%$ \\
\hline
\end{tabular}

Tabela 2 - Distribuição do uso de EPIs pelos enfermeiros consoante os cuidados prestados

Nos contatos diretos com o paciente, os EPIs reduzem o risco de contaminação da farda, devendo ser colocados antes do contato, removidos imediatamente após a sua utilização e de uso único. Só assim, se verifica a proteção dos enfermeiros e dos pacientes da transmissão cruzada de microrganismos provenientes de outros utentes ou ambientes contaminados (Costa, 2017; Fernandes, 2017; Gonçalves, 2014; Santos, 2017; Williams et al., 2015).

Objetivando avaliar a contaminação das fardas clínicas, foram realizadas análises a 72 colheitas de amostras microbiológicas, recolhidas através de zaragatoa em três locais diferentes da farda de cada enfermeiro participante: RA, TD e TE. O dado que nos indica a contaminação da farda clínica corresponde à UFC/ml, que significa Unidade Formadora de Colónia por mililitro. Na tabela 3, encontram-se descritos os resultados das colheitas.

\begin{tabular}{|c|c|c|c|c|c|}
\hline $\begin{array}{c}\text { Grupo de bactérias } \\
\text { encontradas nas fardas }\end{array}$ & $\begin{array}{c}\text { Quantidade de fardas } \\
\text { clínicas com bactérias }\end{array}$ & \multicolumn{3}{|c|}{$\%$ de UFC/ml por localização } \\
\cline { 2 - 5 } & $\mathbf{N}^{\mathbf{0}}$ & $\%$ & RTD & RTE & RA \\
\hline Staphylococcus Aureus & 14 & $58,3 \%$ & $25 \%$ & $20,8 \%$ & $29,2 \%$ \\
\hline $\begin{array}{c}\text { Outras bactérias do grupo } \\
\text { Staphylococcus }\end{array}$ & 23 & $95,8 \%$ & $83,3 \%$ & $87,5 \%$ & $75 \%$ \\
\hline $\begin{array}{c}\text { Bactérias do grupo } \\
\text { Streptococcus }\end{array}$ & 10 & $41,7 \%$ & $20,8 \%$ & $29,2 \%$ & $25 \%$ \\
\hline Acinetobacter baumannii & 2 & $8,3 \%$ & -- & $8,4 \%$ & $4,2 \%$ \\
\hline Outros Bacilos & 10 & $41,7 \%$ & $20,8 \%$ & $12,6 \%$ & $8,4 \%$ \\
\hline
\end{tabular}

Tabela 3 - Resultados das colheitas feitas às fardas clínicas

No que diz respeito à diversidade microbiológica, foram encontradas as bactérias Staphylococcus Aureus, Staphylococcus, Outros Bacilos, Acinetobacter Baumannii e 


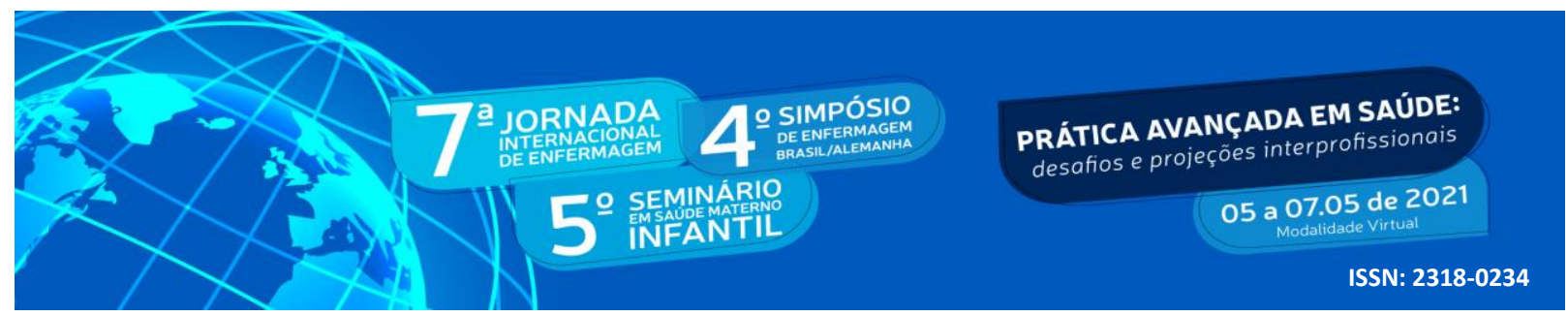

Streptococcus. Estes resultados diferem de outros estudos realizados com adultos (Costa, 2017; Fernandes, 2017; Santos, 2017) em que há uma prevalência não só das bactérias do grupo Staphylococcus, mas também de Enterococcus.

A tipologia das bactérias encontradas pode ser explicada por serem bactérias encontradas predominantemente em afeções dos tratos respiratório (Staphylococcus Aureus, Staphylococcus, Acinetobacter Baumannii e Streptococcus), urinário (Acinetobacter Baumannii), pele e intestino (Streptococcus) que se relacionam com os cuidados pediátricos descritos pelos enfermeiros. Pegar uma criança pequena ao colo para prestar cuidados implica o contato direto da face e tronco com a farda, constituindo-se um vetor de contaminação, através das vias aéreas e pele. Também a incontinência dos esfíncteres urinários e intestinal predispõe à contaminação das fardas clínicas, aquando dos cuidados de higiene ou mudança da fralda. Quando se realizou a colheita de dados deste estudo, havia uma predominância de infeções respiratórias em ambos os serviços e vários enfermeiros referiram pegar ao colo os $\mathrm{RN}$ e crianças pequenas, o que pode ter ocasionado a contaminação das fardas com secreções.

Após a análise das amostras colhidas nas fardas clínicas verificou-se que, em todas as regiões há contaminação microbiológica, sendo as zonas com maior percentagem de microrganismos as regiões RA e TE, o que está alinhado com os enfermeiros serem destros.

No que concerne à relação existente entre a contaminação microbiológica da farda clínica e o tipo de cuidados de enfermagem de contacto prestados ao RN e criança pequena, explanado na tabela 4, verificou-se existir uma relação positiva entre: o método de canguru ou colo para conforto e a presença de bactérias Acinetobacter Baumannii e Streptococcus; o colo (para transporte, contenção, ou outro) e a presença na farda da bactéria Acinetobacter Baumannii e entre outros cuidados prestados pelos enfermeiros e as bactérias do grupo de Outros Bacilos.

\begin{tabular}{|c|c|c|c|}
\hline Cuidados & Bactéria & Qui-quadrado $\left.\mathbf{( X}^{\mathbf{2}}\right)$ & $\begin{array}{c}\text { Significância } \\
\text { Estatística (p) }\end{array}$ \\
\hline $\begin{array}{c}\text { Método canguru ou } \\
\text { colo para conforto }\end{array}$ & Acinetobacter Baumannii & 4,364 & 0,037 \\
\cline { 2 - 4 } & Streptococcus & 5,486 & 0,019 \\
\hline $\begin{array}{c}\text { Colo para transporte, } \\
\text { contenção ou outro }\end{array}$ & Acinetobacter Baumannii & 4,364 & 0,037 \\
\hline Outros cuidados*: & Outros Bacilos & 5,486 & 0,019 \\
\hline
\end{tabular}

*incluem a administração de terapêutica via endovenosa; colheita de espécimes para análise; limpeza nasal com soro fisiológico; transferência para outro serviço; aspiração de secreções 


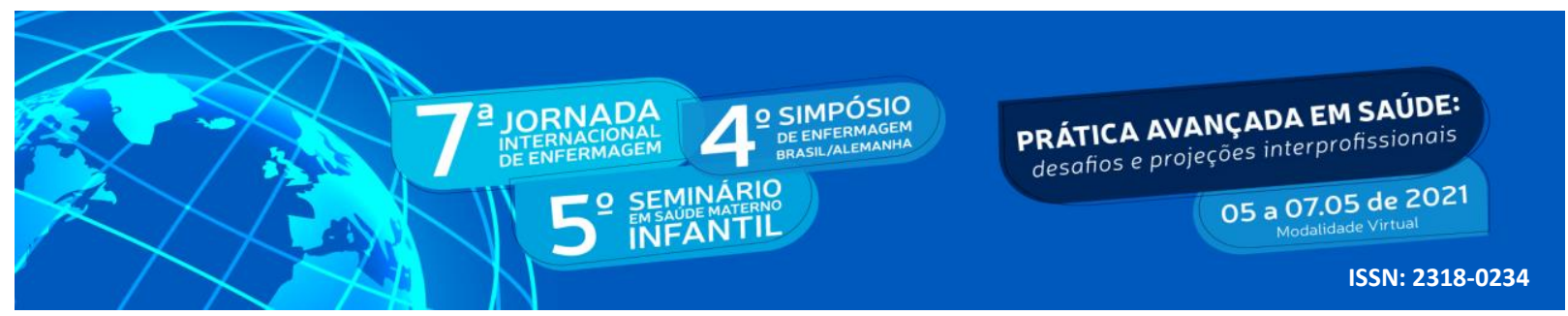

Tabela 4 - Relação entre a contaminação microbiológica da farda e o tipo de cuidados de contacto prestados pelos enfermeiros

Os cuidados de enfermagem "método canguru ou colo para conforto"; "colo para transporte, contenção, ou outro" não são os mais frequentes, no entanto, são os que envolvem maior contato físico direto com os recém-nascidos ou crianças pequenas. Isto é, apenas 33,3\% dos enfermeiros prestaram estes cuidados, contudo, há uma relação estatística significativa entre esses cuidados e a maior contaminação microbiológica da farda clínica.

\section{CONCLUSÃO}

A farda clínica pode ser um veículo de transmissão e de contaminação por microrganismos de elevada relevância epidemiológica, constituindo-se um foco para a disseminação de microrganismos patogénicos por utentes, profissionais e ambientes. Cabe ao enfermeiro gerir o seu vestuário tendo em conta os padrões de qualidade e as medidas de prevenção de contaminação e propagação de microrganismos, e assim, minimizar o risco de infeção.

Dos resultados obtidos neste estudo, o que mais importa destacar é que, apesar de não ter sido encontrada relação estatística significativa entre a gestão da farda clínica e a sua contaminação microbiológica, verificou-se não existir uma correta gestão da farda por parte dos enfermeiros que participaram no estudo. Quanto ao local de higienização da farda, os dados mais relevantes apontam para que apenas 58,3\% dos enfermeiros lavam a farda clínica na lavandaria da instituição de saúde, apesar das recomendações internacionais indicarem que este é o tratamento de roupa seguro. Também o uso de EPIs não é uma prática sistemática dos enfermeiros deste estudo, salientando-se que $70 \%$ dos participantes nunca utilizaram EPI aquando da realização de procedimentos que requerem técnica assética.

Este estudo evidenciou a existência de relações significativas entre alguns dos cuidados de enfermagem de contato direto ao recém-nascido e/ou criança pequena e o risco de contaminação microbiológica com diversas bactérias, nomeadamente o método de canguru ou colo para conforto, o colo para transporte, contenção, ou outro, e ainda entre outros cuidados que incluem 


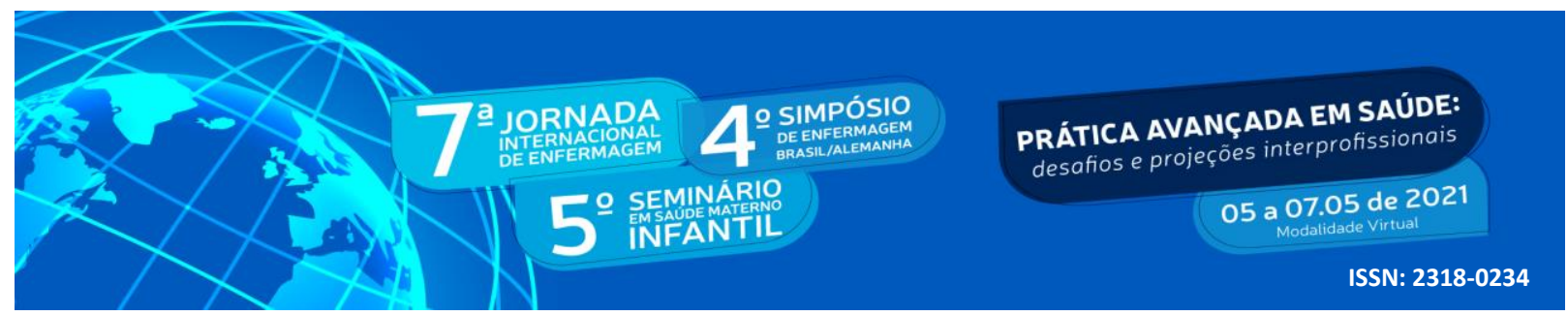

a administração de terapêutica via endovenosa, colheita de espécimes para análise, limpeza nasal com soro fisiológico, transferência para outro serviço ou aspiração de secreções.

Como limitação do estudo, de referir que a amostra é de tamanho reduzido, o que pode justificar não terem sido encontradas mais relações significativas entre as variáveis consideradas. No entanto, esta investigação evidencia a importância e necessidade de mais estudos para aprofundar a prevenção e controlo de infeções associadas aos cuidados de enfermagem em contexto pediátrico, devendo estes se focar não apenas na contaminação da farda, mas também do material de bolso, brinquedos presentes nas salas comuns, bem como a efetividade das medidas de prevenção de infeção.

É urgente a necessidade de uniformizar práticas e comportamentos dos profissionais no que toca à gestão da farda, através de normas concretas elaboradas pelas instituições, formação na área dos profissionais e, evidentemente, garantir o adequado tratamento dos uniformes, de modo a tornar a prática mais segura, tanto para enfermeiros, como para as crianças que são alvo de cuidados.

\section{REFERÊNCIAS}

ANDERSON, Deverick J. et al. The Antimicrobial Scrub Contamination and Transmission (ASCOT) Trial: A Three-Arm, Blinded, Randomized Controlled Trial With Crossover Design to Determine the Efficacy of AntimicrobialImpregnated Scrubs in Preventing Healthcare Provider Contamination. Infection Control \& Hospital Epidemiology, Cambridge, p. 11471154, 26 jul. 2017. DOI 10.1017/ice.2017.181.

BEARMAN, Gonzalo et al. Healthcare Personnel Attire in Non-Operating-Room Settings. Infection control and hospital epidemiology, [S. l.], v. 35, n. 2, p. 107-121, 14 fev. 2014. DOI 10.1086/675066. Disponível em: http://www.jstor.org/stable/10.1086/675066 .

CARVALHO, Eduardo S.; MARQUES, Silvia R. Infeção hospitalar em pediatria. Jornal de Pediatria: Sociedade Brasileira de Pediatria, [s. l.], v. 75, ed. 1, p. 31-45, fev. 1999. DOI 00217557/99/75. Disponível em: http://www.jped.com.br/conteudo/99-75-S31/port.pdf.

COSTA, Paulo Jorge dos Santos. GESTÃo DE MATERIAL CLÍNICO DE BOLSO POR ENFERMEIROS: FATORES DETERMINANTES E AVALIAÇÃO MICROBIOLÓGICA. Orientador: João Manuel Garcia Nascimento Graveto. 2017. 174 p. Dissertação (Mestrado em Enfermagem) - Escola Superior de Enfermagem de Coimbra, Coimbra, 2017. Disponível em: http://web.esenfc.pt/?url=RruTyEuU. 


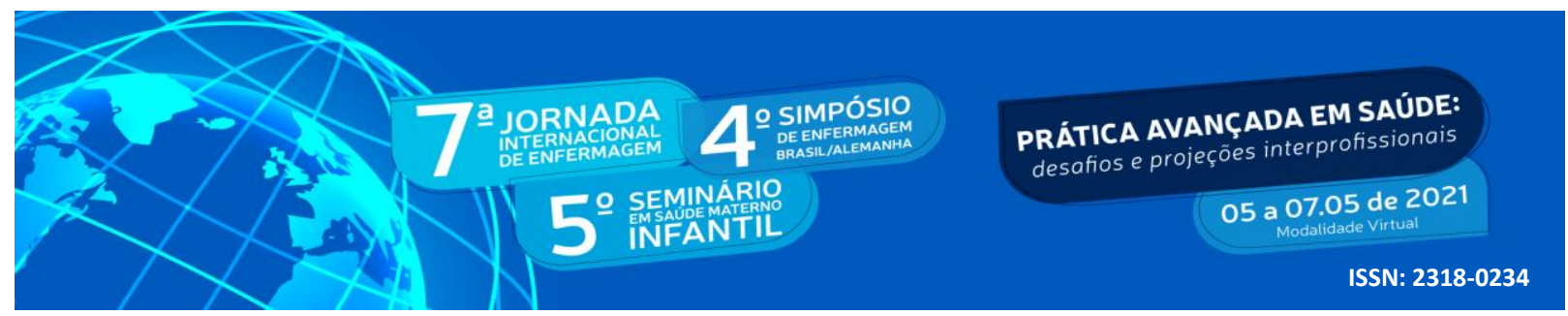

DIREÇÃO-GERAL DA SAÚDE (Portugal). 2013. Norma n029/2012 de 28/12/2012: Precauções Básicas de Controlo da Infeção (PBCI), [S. l.], 2013. Disponível em: https://www.dgs.pt/directrizes-da-dgs/normas-e-circulares-normativas/norman0292012-de28122012-png.aspx.

FERNANDES, Elisabete Amado. Gestão da farda clínica por enfermeiros: fatores determinante e avaliação microbiológica. Orientador: João Manuel Garcia Nascimento Graveto. 2017. 138 p. Dissertação (Mestrado em Enfermagem) - Escola Superior de Enfermagem de Coimbra, Coimbra, 2017. Disponível em: http://web.esenfc.pt/?url=2c0UNqzz.

GONÇALVES, Susana Maria FIlipe. Prevenção e controlo da infeção na prática dos enfermeiros: Contributos da Formação. Orientador: Maria Helena Brísio. 2012. 184 p. Dissertação (Mestrado em Enfermagem) - Escola Superior de Enfermagem de Coimbra, Coimbra, 2012. Disponível em: http://repositorio.esenfc.pt/?url=dKGN2NTh.

INTERNATIONAL SCIENTIFIC FORUM ON HOME HYGIENE INFORMATION. 2013. Clothing, household linens, laundry \& home hygiene, $\left[\begin{array}{ll}S . & l .]\end{array}\right]$ 2013. Disponível em: https://www.ifhhomehygiene.org/system/files_force/publications/Clothing_laundry_and_hom e_hygien e-Oct2013.doc?download=1. Acesso em: 22 mar. 2021.

ARAÚJO, Cláudia Patrícia Machado. Prevenção de Infeções Associadas aos Cuidados de Saúde: Contributo do Enfermeiro Especialista em Enfermagem de Saúde Infantil e Pediátrica na Segurança e Qualidade dos Cuidados. Orientador: Margarida Malcata; Ana Lúcia Ramos. 2019. 242 p. Relatório de Estágio (Mestrado em Enfermagem) - Instituto Politécnico de Setúbal, Setúbal, 2019. Disponível em: http://hdl.handle.net/10400.26/29028.

OLIVEIRA, Adriana Cristina; SILVA, Marlene da Dores; GARBACCIO, Juliana Ladeira. VESTUÁRIO DE PROFISSIONAIS DE SAÚDE COMO POTENCIAIS RESERVATÓRIOS DE MICRORGANISMOS: UMA REVISÃO INTEGRATIVA. Texto \& Contexto Enfermagem, Florianápolis, ano 25, v. 21, ed. 3, p. 684-691, Julho-Setembro 2012. DOI s. Disponível em: https://www.scielo.br/pdf/tce/v21n3/v21n3a25.pdf.

GASPAR, Cristina. GESTÃo DO PROCEDIMENTO HIGIENIZAÇÃO DAS MÃOS POR ENFERMEIROS: FATORES DETERMINANTES E AVALIAÇÃO MICROBIOLÓGICA. Orientador: João Manuel Garcia Nascimento Graveto. 2017. 161 p. Dissertação (Mestrado em Enfermagem) - Escola Superior de Enfermagem de Coimbra, Coimbra, 2017. Disponível em: http://web.esenfc.pt/?url=Mk70EVLU.

SIEGEL, D., RHINEHART, E., JACKSON, M., \& CHIARELLO, L. 2007. Guideline for Isolation Precautions: Preventing Transmission of Infectious Agents in Healthcare Settings. Healthcare Infection Control Practices Advisory Committee, [S. l.], 207. Disponível em: https://www.cdc.gov/infectioncontrol/pdf/guidelines/isolation-guidelines-H.pdf.

TIPPLE, Anaclara; PEREIRA, Milca; HAYASHIDA, Miyeco; MORIYA, Tokico; SOUZA, Adenícia. O ensino do controle de infecção: um ensaio teórico-prático. Revista Latino Americana da Enfermagem, Ribeirão Preto, v. 11, ed. 2, Março-Abril 2003. Disponível em: http://dx.doi.org/10.1590/S0104-11692003000200017. 


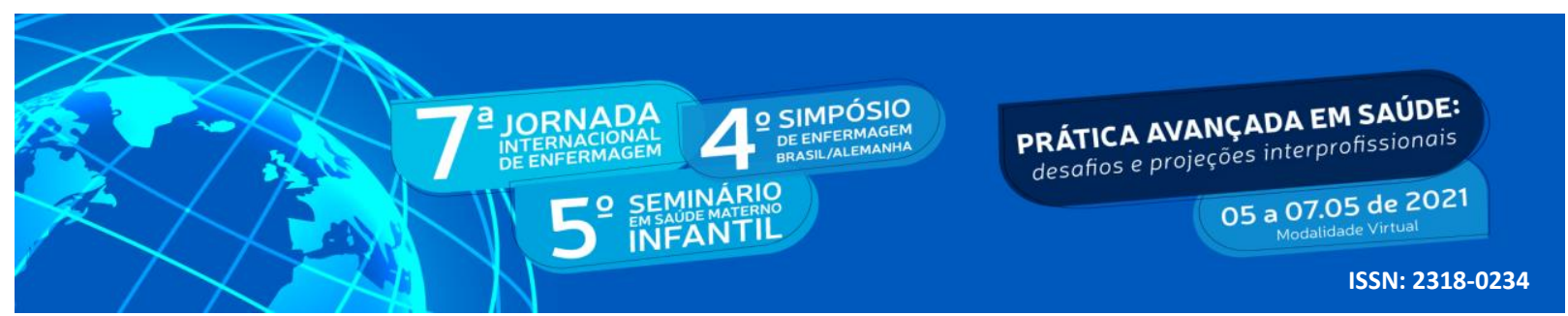

VALADARES, Bruno; BARBOSA, Railane; TEIXEIRA, Raquel; OLIVEIRA, Rodrigo; TOMICH, Geórgia. Contaminação de uniformes privativos utilizados por profissionais que atuam nas unidades de terapia intensiva. Revista de Epidemiologia e Controle da Infeção, [s. l.], 2017. DOI http://dx.doi.org/10.17058/reci.v7i1.7380.

WILLIAMS, Calvin et al. Impact of universal gowning and gloving on health care worker clothing contamination. Infection Control \& Hospital Epidemiology, [s. l.], v. 36, ed. 4, p. 431-437, 2015. DOI 10.1017/ice.2014.75. Disponível em: https://pubmed.ncbi.nlm.nih.gov/25782898/. 